\title{
Formación en la Empresa Universitaria: Centro Organizador de Congresos y Convenciones
}

\author{
Pablo Szmulewicz Espinosa ${ }^{1}$ \\ Cecilia Gutierrez Vega ${ }^{2}$
}

RESUMO: Trata do Centro Organizador de Congressos e Convenções do Instituto de Turismo da Universidad Austral de Chile, considerando que a prática contribui para a melhor fornação dos educandos. Aborda a origem e o desenvolvimento desse Centro, as áreas em que atua, os serviços que oferece e sua estrutura. Destaca a relação desses serviços com a formação universitária em Turismo.

PALAVRAS-CHAVE: Turismo e eventos; organização de eventos; fornação universitária; Universidad Austral de Chile.

ABSTRACT: The Centre Organizer of Congress and Conventions of the Institute of Tourism is presented. Whereas the practicedocontributes to the best background of the students. For such, the text points out relations about the centre's origination and development, its performance's cantent, services which are offered and its operacional structure. Further, the paper eniphasizes the relationship between these services and the graduated degree of tourism education.

KEY WORDS: Tourismand events; event's organization, graduated degree of tourism education; Universidad Austral de Chile.

1. Académico del Instituto de Turismo de la Escuela de Turismo - Universidad Austral de Chile.

2. Estudiante del Instituto de Turismo de la Escuela de Turismo - Universidad Austral de Chile.

End. para corresp.: Instituto de Turismo de la Universidad Austral de Chile. Valdivia - Chile. Fax (63) 21-1158. 


\section{Origen y Desarrollo}

La actual gestión de la Escuela de Administración de Empresas Turisticas de la Universidad Austral de Chile se orienta por los siguientes objetivos estratégicos:

- actualización del plan de estudios;

- separación formación profesional - Licenciatura;

- incorporación actividades prácticas.y giras, al plan;

- vinculación con empresas universitarias;

- creación Hotel-Escuela/Agencia de Viajes/O.P.C.;

- implementación Talleres - laboratorios alimentos y bebidas, agencias de viajes, planificación turística;

- participación en redes universitarias de cooperación para intercambioacadémico y estudiantil.

En este marco se inserta la creación y desarrollo de una empresa universitaria Organizadora de Congresos y Convenciones, por parte del Instituto de Turismo.

El Centro Organizador de Congresos y Convenciones dependiente del Instituto de Turismo de la Universidad Austral de Chile, nace en 1989, dando a conocer los servicios que se ofrecen y atendiendo algunos eventos. En 1991 su director, prof. Pablo Szmulewicz Espinosa assume la responsabilidad de desarrollar el Centro y proyectarlo tanto al interior como fuera de la Universidad. Entre 1991 y 1997 se han atendido los siguientes eventos:

- Primeras Jornadas sobre Formación y Capacitación de Recursos Humanos para la Actividad Turistica - Mayo de 1991;

- Curso Internacional sobre Planificación Turistica - Noviembre de 1991;

- Primer Congreso Chileno de Ornitología - Septiembre de 1991;

- Novenas Jornadas Nacionales e Intenacionales de Odontología - Noviembre de 1991;

- Primera Escuela de Verano sobre Relaciones Internacionales - Enero de 1992;

- Simposio Internacional de Manejo Forestal - Marzo de 1993;

- Cena de Pan y Vino - Agosto de 1993;
- Seminario de Planificación Turística - Diciembre de 1993;

- Reunión Internacional CAMCORE (Cooperativa de Mejoramiento Genético) Noviembre/Diciembre de 1993;

- Curso "Diseño de Planta, Selección y Distribución de Equipos para la Industria de Alimentos" - Junio de 1994;

- Curso “Calidad Integral de las Empresas Turisticas” - Diciembre de 1994;

- Curso de Informadores Turísticos - Junio de 1995;

- Curso "Desarrollo Turístico a Nivel Comunal" - Septiembre de 1995;

- Conferencia Sr. Secretario General de la OMT - Junio de 1995;

- IX Congreso Nacional de Cámaras de Turismo, Pucón - Septiembre de 1995;

- Curso Internacional “Administración de Empresas de Servicios Gastronómicos” - Octubre de 1995;

- XIII Encuentro Nacional de Facultades de Administración y Economía - Enero de 1996;

- Primer Seminario Nacional de Agroturismo y Turismo Rural, Frutillar - Marzo de 1996;

- Seminario Técnico Ecoturismo y Turismo de Aventura - Septiembre de 1996;

- Seminario Internacional Agroturismo y Turismo Rural, Olmué - Abril de 1997.

La trayectoria del Centro muestra que las actividades han ido en permanente aumento de complejidad partiendo desde eventos del Instituto hasta pasar a organizar eventos de otras unidades de laUniversidad, incluso de otras instituciones y organizados fuera de Valdivia. En estos eventos han trabajado más de 50 estudiantes y el trabajo del Centro ha recibido elogiosos comentarios de todos sus "clientes". Merece destacarse especialmente el reconocimiento que ha recibido de parte de Federación Nacional de Cámaras de Turismo de Chile.

En el marco de los fructíferos programas de intercambio académico que posee la Universidad Austral, esta experiencia fue transmitida a la Universidad de las Américas de Puebla, México, en una pasantia del Director del Centro a principios de 1994. Durante la estadia de tres meses como profesor visitante organizó el $4^{\circ}$ Congreso Nacional de Hotelería en Puebla, en Abril de 1994, con la colaboración de 15 estudiantes de la Licenciatura en Hoteleria de esa Universidad. 


\section{Areas de Acción, Servicios Ofrecidos y Estructura}

Las areas de acción del Centro son:

- prácticas profesionales: hasta la fecha tres estudiantes han realizadoparte de sus prácticas profesionales en la Unidad, desarrollando actividades durante varios meses;

- servicios a terceros del Organización del eventos: el Centro ofrece toda su gama de servicios a distintas unidades de la Universidad e incluso a otras instituciones, tanto en forma integral como en forma parcial;

- investigación aplicada: el Centro ha impulsado el desarrollo de investigaciones acerca del tema de los Congresos y Convenciones tanto a nivel nacional como regional. Parte de estos estudios se han publicado en revistas nacionales e internacionales:

- tesis en el ámbito de la temática de los eventos: diversas tesis de la carrera de Administración de Empresas Turísticas se han realizado bajo el alero del Centro y utilizando como base la experiencia y los contactos del Centro: Estrategia de Promoción de Congresos en Valdivia; Los Congresos y Convenciones Internacionales en Chile; Manual de Procedimientos para la Organización de Congresos y Convenciones; Producción de Exposiciones; Factibilidad de una Empresa Organizadora de Congresos en Valdivia, son algunos títulos;

- prácticas estudiantiles: más de 50 estudiantes de la carrera de Turismo han desarrollado experiencias prácticas en el Centro

- vinculacióninstitucional (OPCE; Cámaras de Turismo; Oficina de Convenciones de Valdivia): a través del Centro se han logrado importantes vinculaciones con la Asociación deOrganizadores Profesionales de Congresos de Chile, la Federación Nacional de Cámaras de Turismo y la Oficina de Convenciones de Valdivia.

El Centro ofrece servicios específicos o el servicio integral de Organización de un Congreso, Convención, Seminario, Curso y toda clase de eventos técnicos, científicos y comerciales:

- planificación del evento, organización del evento, difusión y promoción, documentación, transporte, recursos humanos, finanzas, equipos de apoyo, ceremonial y protocolo, secretaría, gestión dereservas de alojamiento y transporte, entrega de información, programa deactividades sociales, programadeactividades culturales, programa de actividadesturísticas, programa de acompañantes, gestión de auspicios y patrocinios, servicio decafetería, programa post evento, exposiciones, entre otros.
El Centro tiene un funcionamiento temporal. Se conforma especialmente para cada evento solicitado. En forma permanente sólo participan de él el Directo y la Secretaria y en carácter de asesoría la oficina de administración y finanzas de la Faculdad de Ciencias Económicas de la Universidad Austral de Chile.

Los estudiantes de cursos superiores con experiencia en el rubro actúan a nivel de jefaturas de comités y Coordinación General, mientras que los estudiantes de cursos inferiores participan como asistentes de los distintos comités.

Excepcionalmente participan en la actividad del Centro otros profesionales y técnicos cuyas calificaciones son diferentes a las de los administradores de empresas turísticas: secretaria, periodistas.

Por otra parte, se subcontratan los servicios profesionales especializados: publicistas, diseñadores, técnicos en sonido, etc.

El Centro trabaja en forma coordinada con agencias de viajes, hoteles, restaurantes, empresas de transporte, empresas banqueteras, entre otras (Figura 1).

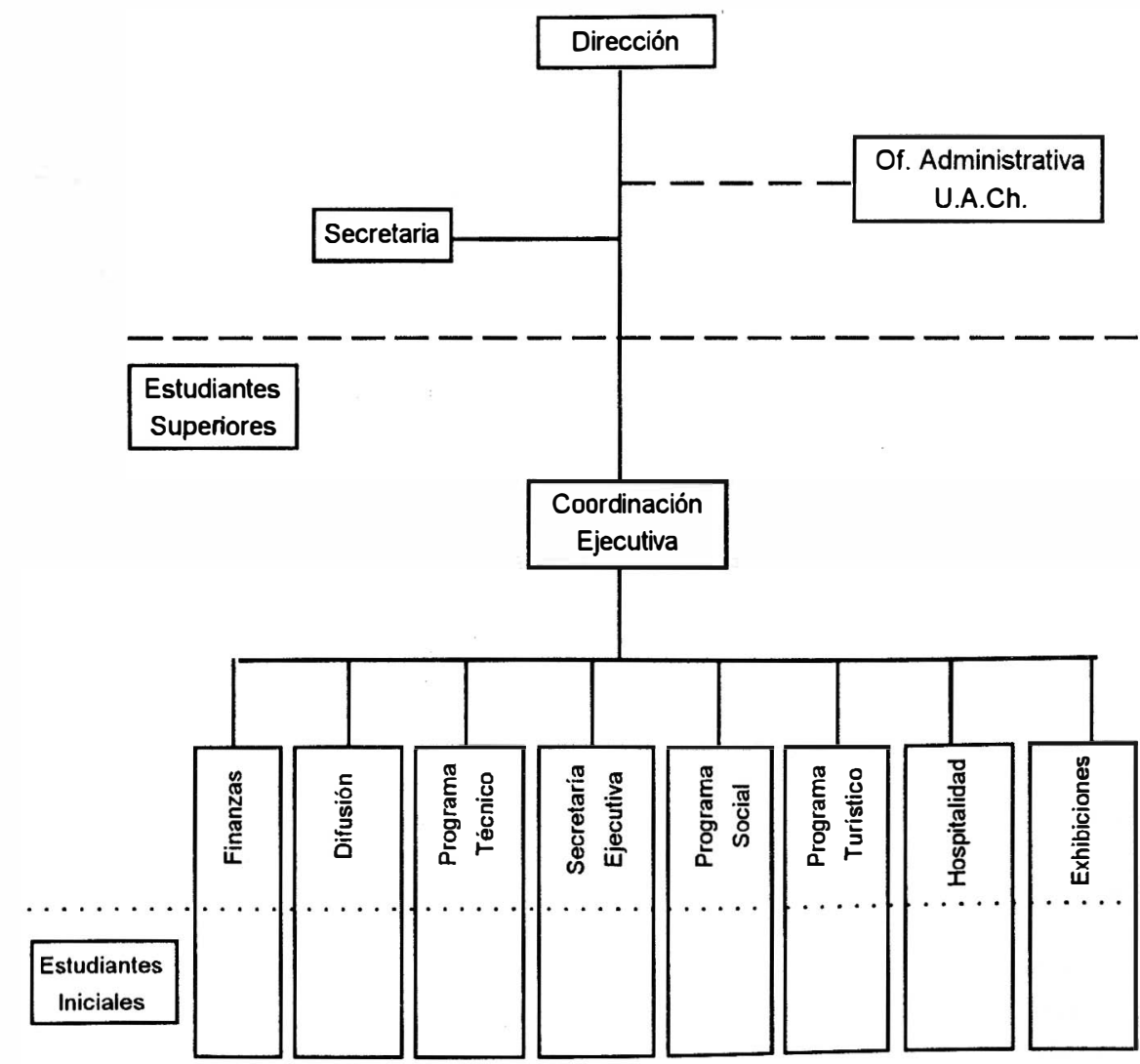

FIGURA I - ESTRUCTURA DEL CENTRO 


\section{Prestación de Servicios y Formación}

La Organización de un evento requiere la aplicación del proceso administrativo al desarrollo de la actividad. En cada etapa del proceso se realizan múltiples tareas y todas ellas se desarrollan en forma coordinada y tratando de lograr el mayor conocimiento y comprensión de parte de todos los participantes de cada una de las decisiones y acciones que se realizan. Sin pretender ser exhaustivo algunas de las tareas principales se presentan en la Figura 2.

Los objetivos formativos del Centro son:

- conocer procesos y técnicas de la organización de convenciones;

- desarrollar habilidades prácticas en la organización de eventos;

- desarrollar habilidades de trabajo en equipo;

- desarrollar cualidades personales: responsabilidad, seguridad, etc.

- establecer una estrecha relación con los contenidos teóricos de la carrera.

Los beneficios para los estudiantes son:

- conocimientos prácticos de organización de eventos;

- prácticas profesionales;

- relaciones profesionales para prácticas y empleos;

- viajes;

- salarios;

- liderazgo, desarrollo personal y trabajo en equipo;

- integración social;

- conocer que es turismo;

- conocimientos acerca de temas diversos:

- desarrollo de la creatividad.

Sin duda el desarrollo del Centro representa importantes esfuerzos, tanto para la dirección como para los estudiantes. Estos últimos deben ocupar gran cantidad de tiempo que restan a sus obligaciones personales y académicas. Por otra parte, participar en una empresa "real" significa enfrentar situaciones de mucha tensión y responsabilidad, además de todas las dificultades propias de trabajar con otras personas y con "clientes" diversos y públicos variados.

En la práctica de organización de eventos se aplican conocimientos de muchas cátedras, como:

- Computación;

- Marketing y Promoción;

- Ventas;

- Administración;
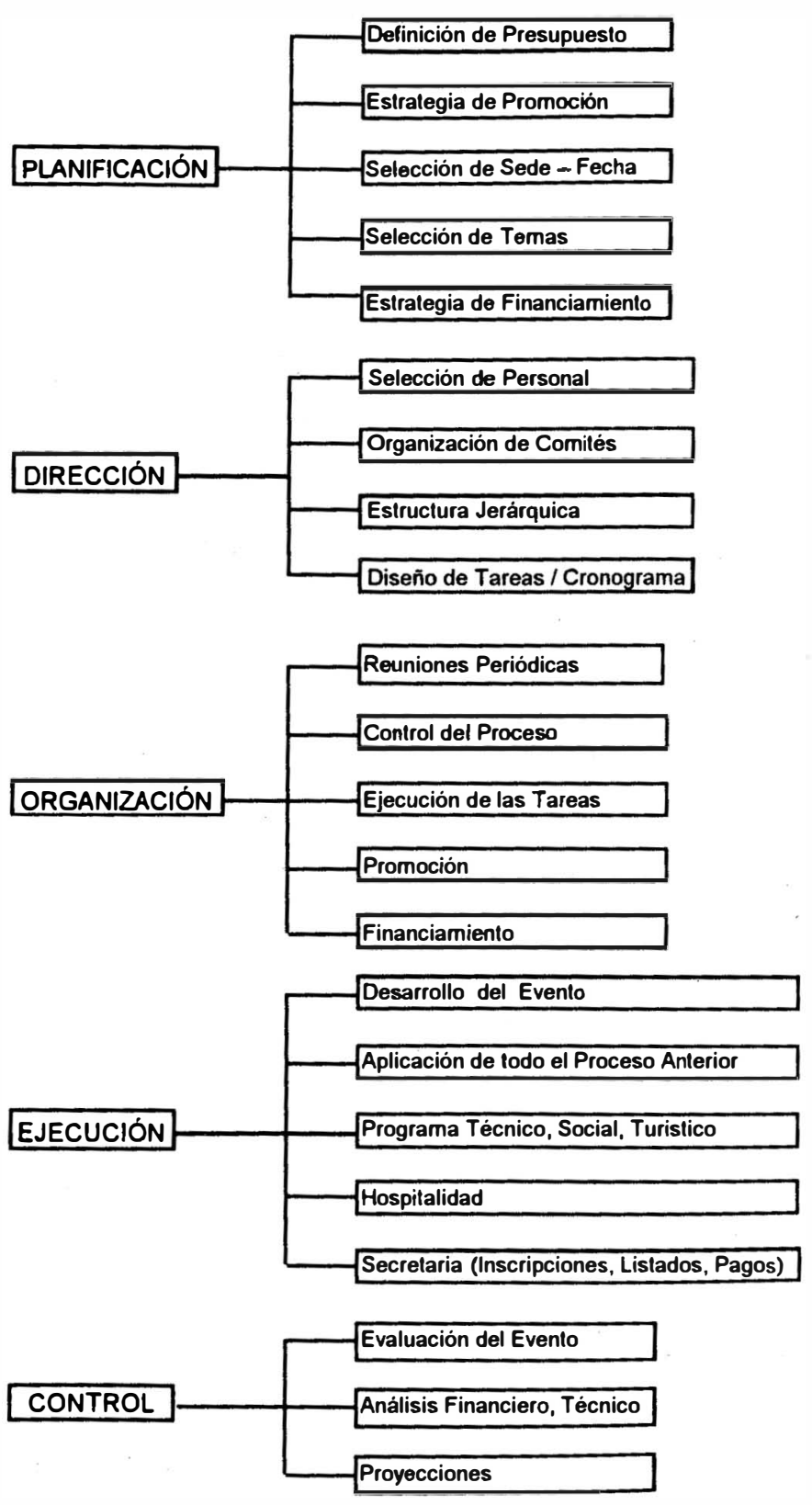

FIGURA 2 - TAREAS PRINCIPALES DEL PROCESO ADMINISTRATIVO 
- Relaciones Humanas;

- Eventos;

- Guía de Turismo;

- Relaciones Públicas

- Idiomas;

- Agencias de Viajes.

De todos los estudiantes que han participado en las actividades del Centro, algunos han realizado todo el camino desde los puestos más bajos de la empresa hasta los más elevados, e incluso han hecho de esta especialidad su opción profesional

Hoy varios titulados se desempeñan en Centros de Convenciones o en Hoteles en el área de Eventos.

Una síntesis de la expresiones de estos estudiantes es la siguiente:

(...) el haber participado en la Unidad de Eventos ha sido una de mis mejores experiencias, he aprendido gran cantidad de conocimientos, muy diversos, que van desde lo referente a las distintas áreas de la organización, hasta desarrollo profesional y personal (Cecilia Gutiérrez V., $4^{\circ}$ Año Adm. de Emp. Tcas., Universidad Austral de Chile).

Me ha servido para desarrollar mi persona y darme cuenta que soy capaz de hace cualquier cosa si me lo propongoy meesfuerzo (Carolina Arancibia, $4^{\circ} \mathrm{Año}$ Adm. de Emp. Tcas., Universidad Austral de Chile).

(...) se puede interiorizar en el conocimiento de organización del cual se pueden saca pautas para organizar cualquier otro tipo de cosas, incluso el tiempo de uno (Graciela Carrizo, $4^{\circ}$ Año Adm. de Emp., Universidad Austral de Chile, alumna procedente de Esquel).

La organización de eventos incentiva el trabajo en equipo, ya que el éxito del evento dependerá del éxito de cada una de las partes que intervienen en la organización (Maritza Guarda, $3^{\circ}$ Año Adm. de Emp. Tcas., Universidad Austral de Chile).

Aprender a abarcar una actividad considerando riesgos e imponderables sin dejar al azar ningün punto. Aprender a adoptar una actitud y una postura profesional frente a situaciones con alto grado de presión (Angélica Cárdenas, Egresada, Adm. de Emp. Tcas., Universidad Austral de Chile).

Participar en esta unidad de eventos me dio la experiencia y conocimientos necesarios para, porqué no, en un futuro, trabajár en ello (Valentina Pérez, Egresada, Adm. de Emp. Universidad Austral de Chile).
(...) dentro de los principales beneficios está la capacidaddeexpresión yel desenvolvimento frente a situaciones laborales, capacidad de conversary llevar acciones por teléfono, que no es fácil, se desarrolla la capacidad de escritura a través de reacción de cartas, informes, etc. (Cristian Sepúlveda, $4^{\circ}$ Año, Adm. de Emp. Tcas., Universidad Austral de Chile)

\section{Conclusiones}

La formación en la práctica constituye una de las mejores modalidades para lograrmás allá de una formación profesional el desarrollo integral de los educandos

El desarrollo de una Empresa Universitaria estructurada en base a docentes y estudiantes requiere de una gran colaboración de parte de estos últimos. Sin ellos en este Centro Organizador de Eventos no habría sido posible realizar todas las tareas encomendadas. La experiencia de cooperación docentes-estudiantes en el trabajo permite establecer nexos que en el aula no es posible cultivar y entregar los estudiantes modelos profesionales que hacen realidad los conocimientos teóricos

La seguridad personal que otorga la participación en experiencias prácticas resulta un elemento de motivación insustituible para avanzar en la formación.

\section{Recebido em 21/5/97}

Aprovado em 10/6/97 